A SEMICLASSICAL, DYNAMICAL MODEL OF THE WATER MOLECULE

Donald Greenspan

Mathematics Department

University of Texas at Arlington

Technical Report \#303 


\title{
A SEMICLASSICAL, DYNAMICAL MODEL OF THE WATER MOLECULE
}

\author{
Donald Greenspan \\ Mathematics Department \\ University of Texas at Arlington
}

Technical Report \#303

\begin{abstract}
A model of the ground state water molecule is formulated dynamically and studied by computer simulations. Excellent approximations of NMR determined bond angles and bond lengths are achieved through electron pairing and simulation of ice $I$.

1. Introduction. The past 75 years has seen an immense amount of research directed at the most fundamental molecule in living matter, the water molecule ${ }^{(1-3)}$. And yet, from both the classical and quantum mechanical points of view, many fundamental questions remain unanswered ${ }^{(1-4)}$. For a single water molecule, only static models are available, and these include those of Bader; Bernal; Duncan and Pople; Ellison and Shull; Gillespie; Heath and Linnett; Rowlinson; and Vernay $(1,5,6)$. Since a dynamical simulation of the water molecule quantum mechanically would require the exact or approximate solution of Schrodinger's equation for a wave function in 39 space dimensions and one time dimension, we will begin more simply in this paper by studying a semiclassical model. The keys to our excellent results will be the introduction of electron pairing and the use of a crystalline state.
\end{abstract}

2. General Model Formulation. Consider a ground state water molecule $M_{1}$ whose oxygen nucleus is denoted by $0_{1}$. Let $M_{2}, M_{3}, M_{4}, M_{5}$, with respective oxygen nuclei $0_{2}, 0_{3}, 0_{4}, O_{5}$, be four neighboring molecules of $M_{1}$. Assume that $M_{1}-M_{5}$ are water molecules in ice $I$, thus allowing us to avoid the complex motions of the liquid state and allowing the introduction of available experimental results into the model ${ }^{(1,3,7)}$.

For the crystal structure of ice $I$, we assume that $O_{2}-0_{5}$ are at the vertices of a regular tetrahedron with $0_{1}$ at the centroid, as shown in Figure 2.1. In each $M_{i}$, let the two hydrogen nuclei be $H_{i j}, i=1,2,3,4,5 ; j=1,2$. Experimentally $(1,2,3,7)$, then, the distance from $\mathrm{O}_{1}$ to each of $\mathrm{O}_{2}, \mathrm{O}_{3}, \mathrm{O}_{4}, \mathrm{O}_{5}$ is $2.76 \AA$ and on each line joining $\mathrm{O}_{1}$ to $0_{2}, 0_{3}$, $\mathrm{O}_{4}, \mathrm{O}_{5}$ there is exactly one $H_{i j}$, one possible such arrangement being that shown in Figure 2.1.

It will be important to recall that the ground state energy $E$ of $M_{1}$ is ${ }^{(8,9,10)}$

$$
E=-(3333.04) 10^{-12} \mathrm{erg} .
$$



simulated.

We now concentrate primarily on $M_{1}$, whose vibrational motions will be

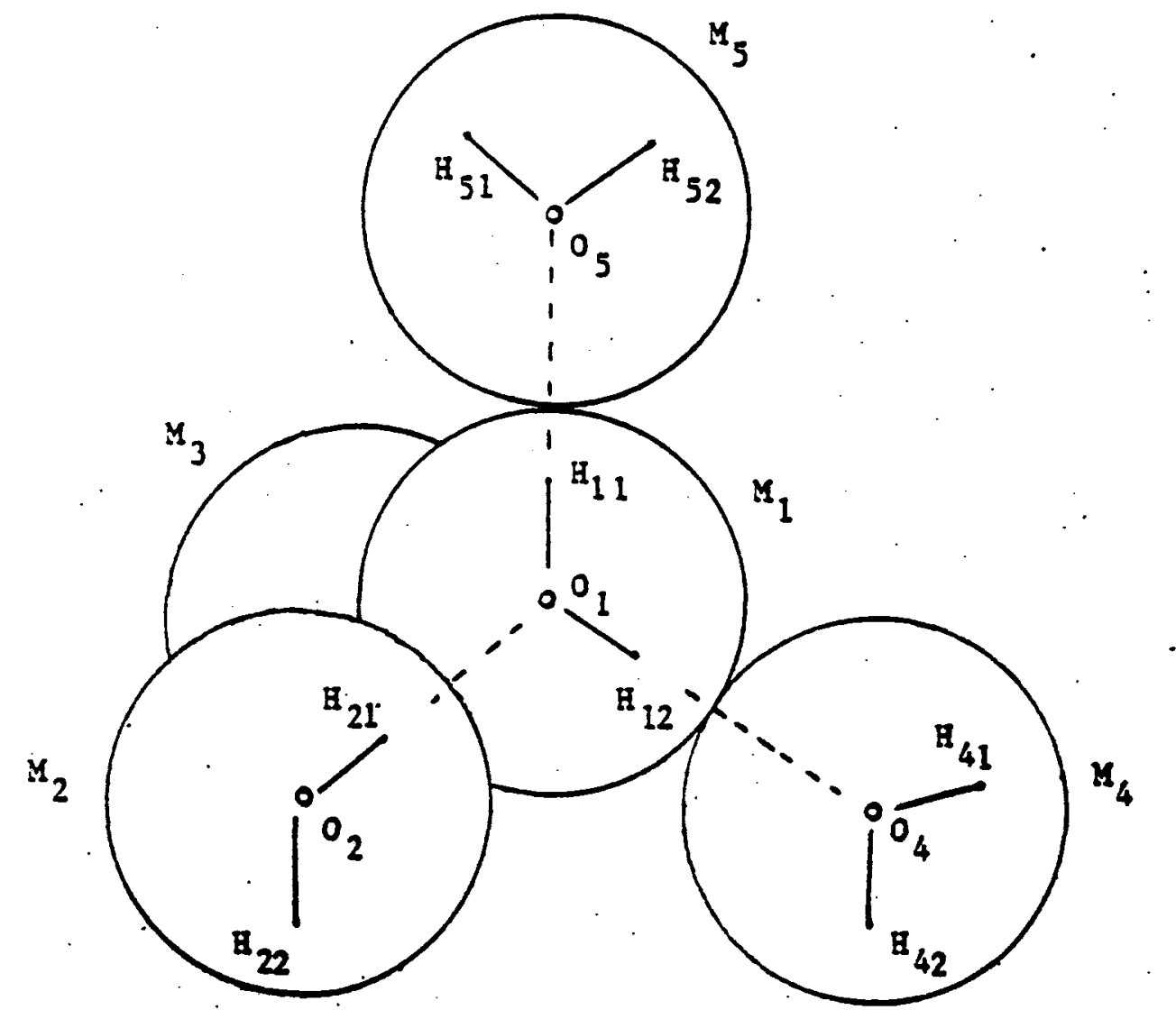

Figure 2.1 
First, let us approximate the Coulombic effect of $M_{2}-M_{5}$ on $M_{1}$. For this purpose, consider then the effect of $M_{2}$ on $M_{1}$. Let $M_{2}$ be a static Duncan and Pople molecule, as shown in Figure 2.2. (Other possible static models will be discussed in Section 6.) Let $Q_{1}$ be the oxygen nucleus; $Q_{2}, Q_{3}$ the protons; and $Q_{4}-Q_{7}$ negative charges. In terms of $e=(4.8028) 10^{-10}$ esu, the Duncan and Pople model assigns charges $C\left(Q_{i}\right)$ by

$$
\begin{gathered}
C\left(Q_{1}\right)=6 e, \quad C\left(Q_{2}\right)=C\left(Q_{3}\right)=e \\
C\left(Q_{4}\right)=C\left(Q_{5}\right)=C\left(Q_{6}\right)=C\left(Q_{7}\right)=-2 e .
\end{gathered}
$$

Now set a hypothetical particle of unit charge $e$ at $Q_{8}=(2.18,0,1.69)$, in the coordinate system of Figure 2.2, so that the distance from $Q_{1}$ to $Q_{8}$ is $2.76 \AA$. Then, the effective Coulombic force of $Q_{1}-Q_{7}$ on $Q_{8}$ is, approximately, $0.06 e^{2}(10)^{16}$. Thus, we replace $M_{2}$ by a single fixed point charge at $O_{2}$ of $C_{1}=0.45 e$. Similarly, $M_{3}$ is replaced by a single point charge at $\mathrm{O}_{3}$ whose charge is $0.45 e$. Analogous calculations allow us to replace $M_{4}$ and $M_{5}$ by point charges at $0_{4}$ and $0_{5}$ whose charges are $C_{2}=-0.23 e$.

As shown in Figure 2.3, the oxygen nucleus $0_{1}$ and the four fixed charges which replace $M_{2}-M_{5}$ are taken to be a motionless, rigid skeletal structure, into which the protons and electrons of $M_{1}$ will be inserted and allowed to move freely, constrained only by conservation of system energy. The coordinates of the $0.45 e$ and $-0.23 e$ charges will be taken to be $(0,2.25,-1.59),(0,-2.25,-1.59),(2.25,0,1.59),(-2.25,0$, 1.59 ), which are the vertices of a regular tetrahedron with edge length 4.5 whose circumscribed sphere has radius 2.76 .

3. Computer Model Formulation. Using the results of Section 2, we proceed now to formulate equations of motion for our water molecule model. We proceed in a new notation which is most convenient for digital computer simulation. In addition, we will introduce changes of variables which simplify the numerical simulation of related initial value problems.

Consider the particles $P_{1}-P_{17}$ shown in Figure 3.1. $P_{1}$ is an oxygen nucleus, $P_{2}$ and $P_{3}$ are protons, $P_{4}-P_{11}$ are electrons, $P_{12}$ and $P_{13}$ are the two first ring electrons of $P_{1}$, and $P_{14}-P_{17}$ are the point charges which are replacing $M_{2}-M_{5}$. In cgs units, for $i=1,2, \ldots, 17$ and at any time $t$, let $P_{i}$ be located at $\vec{r}_{i}=\left(x_{i}, y_{i}, z_{i}\right)$, have velocity $\vec{v}_{i}=\left(\dot{x}_{i}, \dot{y}_{i}, \dot{z}_{i}\right)$ and have acceleration $\vec{a}_{i}=\left(\ddot{x}_{i}, \ddot{y}_{i}, \ddot{z}_{i}\right)$.

Quantum mechanically, the Ehrenfest equations, which are Newtonian dynamical equations for expectation values, are always valid ${ }^{(1)}$. Moreover, when the wave function is narrow, Newton's equations themselves provide valid dynamical approximations over short time periods ${ }^{(11-13)}$. We proceed then under assumptions which allow us to employ Newton's equations for short time periods. Since $P_{1}$ and $P_{14}-P_{17}$ are fixed for all time, the classical equations of motion for the moving particles $P_{i}, i=2,3, \ldots, 13$, are

$$
m_{i} \vec{a}_{i}=\sum_{\substack{j=1 \\ j \neq i}}^{17} \frac{e_{i} e_{j}}{r_{i j}{ }^{2}} \frac{\vec{r}_{j i}}{r_{i j}}, i=2,3, \ldots, 13,
$$




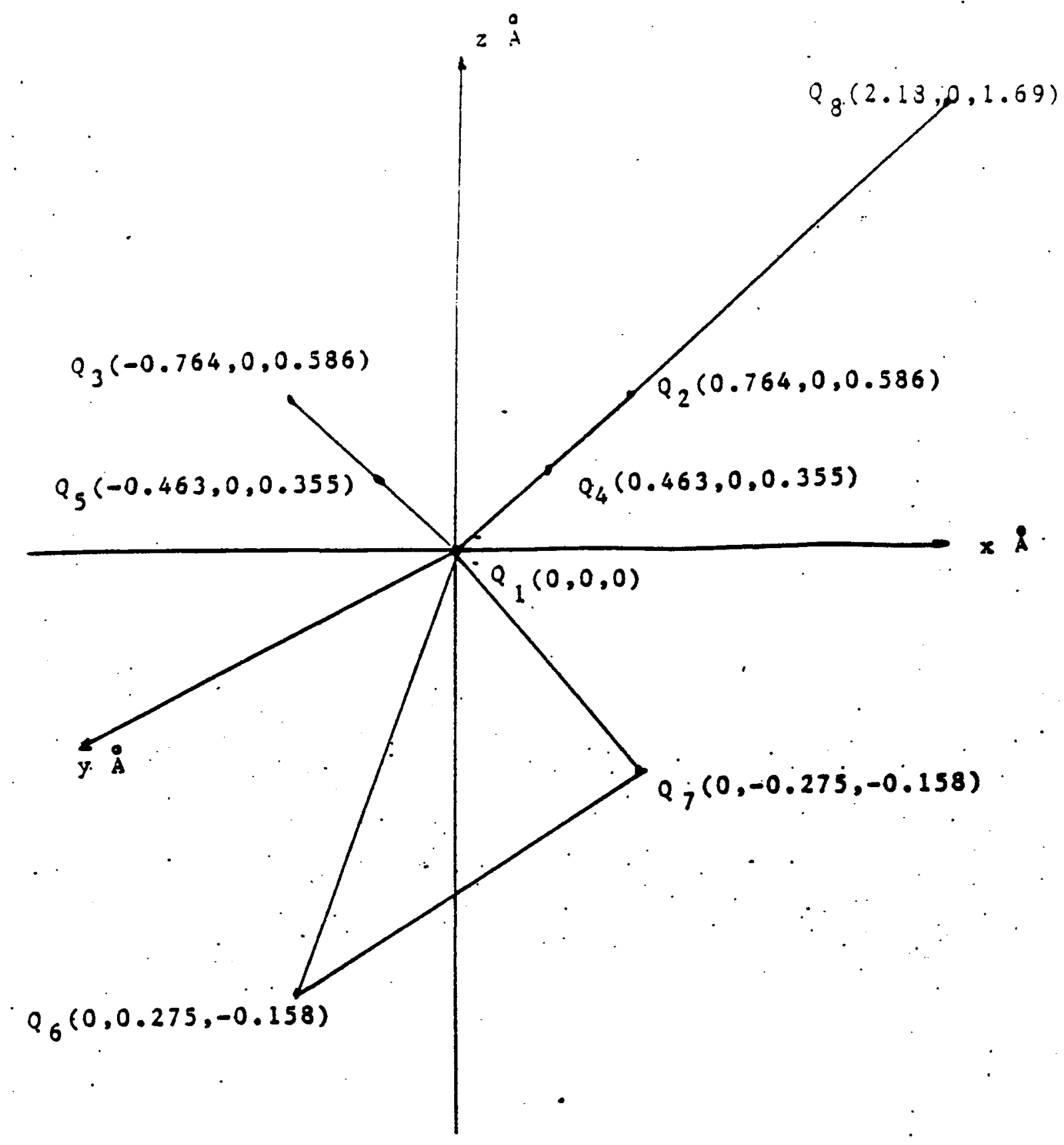

Figure 2.2 


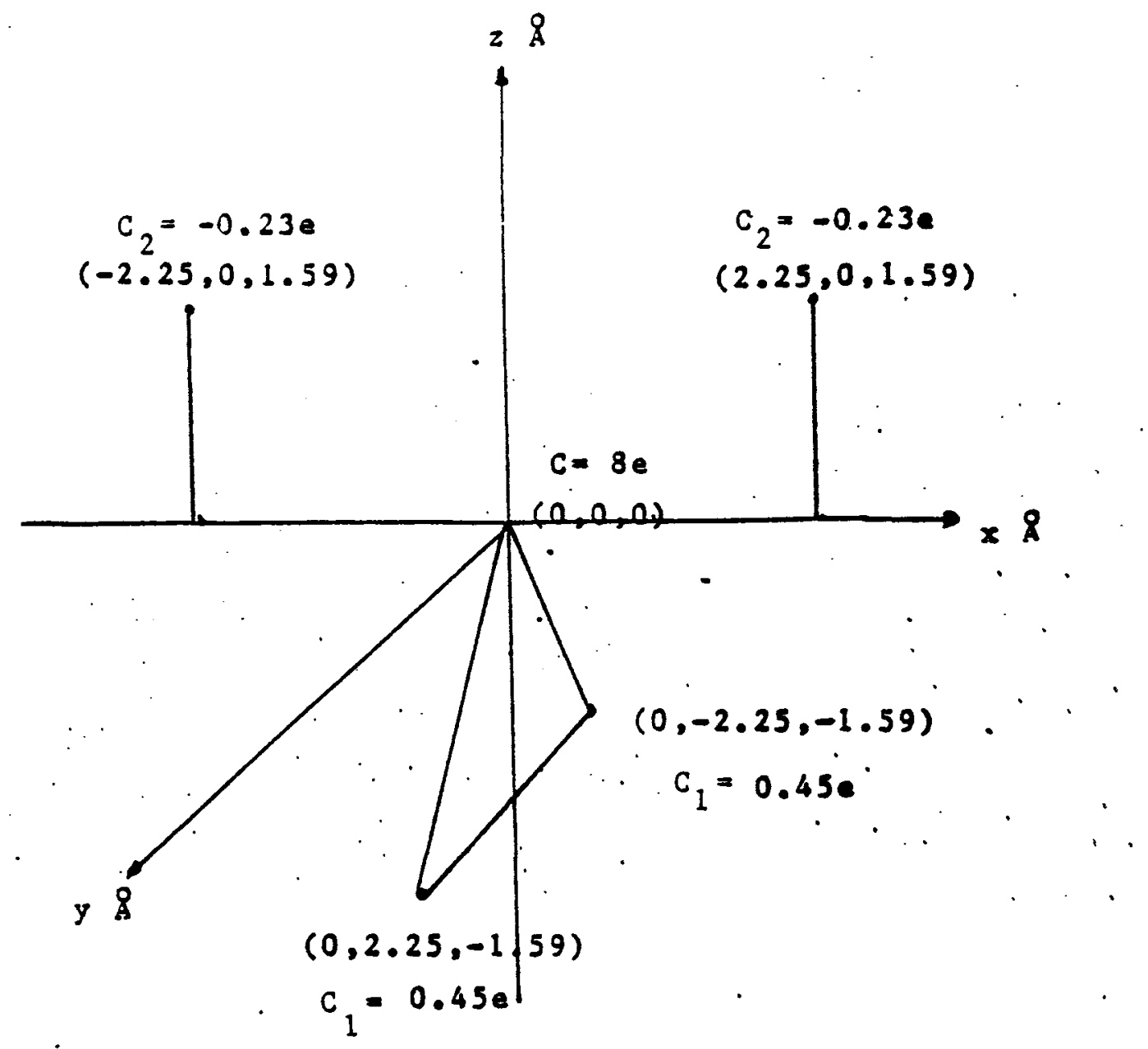

Figure 2.3 
in which $\vec{r}_{j i}$ is the vector from $P_{j}$ to $P_{i}, r_{i j}=r_{j i}=\left\|\vec{r}_{j i}\right\|$, the mass $m_{i}$ and the charge $e_{i}$ of each $P_{i}, i=1,2, \ldots, 13$, are

$$
\begin{aligned}
& m_{1}=16(16724) 10^{-28} \mathrm{gr} \\
& m_{2}=m_{3}=(16724) 10^{-28} \mathrm{gr} \\
& m_{4}=m_{5}=\ldots=m_{13}=(9.1085) 10^{-28} \mathrm{gr} \\
& e_{1}=8(4.8028) 10^{-10} \mathrm{esu} \\
& e_{2}=e_{3}=(4.8028) 10^{-10} \mathrm{esu} \\
& e_{4}=e_{5}=\ldots=e_{13}=-(4.8028) 10^{-10} \mathrm{esu},
\end{aligned}
$$

and,

$$
\begin{aligned}
& e_{14}=e_{15}=.45(4.8028) 10^{-10} \mathrm{esu} \\
& e_{16}=e_{17}=-.23(4.8028) 10^{-10} \mathrm{esu} .
\end{aligned}
$$

From given initial data, the time invariant ground state energy must always satisfy

$$
-(3333.04) 10^{-12}=\frac{1}{2} \sum_{i=2}^{13} m_{i} v_{i}^{2}+\sum_{j=i+1}^{17} \sum_{i=1}^{16} \frac{e_{i} e_{j}}{r_{i j}} .
$$

We now make some convenient changes of variables. First set

$$
T=10^{22} t, \vec{R}_{i}=10^{12} \vec{r}_{i},
$$

in which $\vec{R}_{i}=\left(X_{i}, Y_{i}, Z_{i}\right)$. Then,

$$
\begin{aligned}
& \vec{V}_{i}=\frac{d \vec{R}_{i}}{d T}=\left(\frac{d X_{i}}{d T}, \frac{d Y_{i}}{d T}, \frac{d Z_{i}}{d T}\right)=10^{-10} \vec{v}_{i} \\
& \vec{A}_{i}=\frac{d \vec{V}_{i}}{d T}=10^{-32} \vec{a}_{i} .
\end{aligned}
$$

If, in addition, we set

$$
M_{i}=m_{i} 10^{28}, E_{i}=e_{i} 10^{10}(4.8028)^{-1},
$$




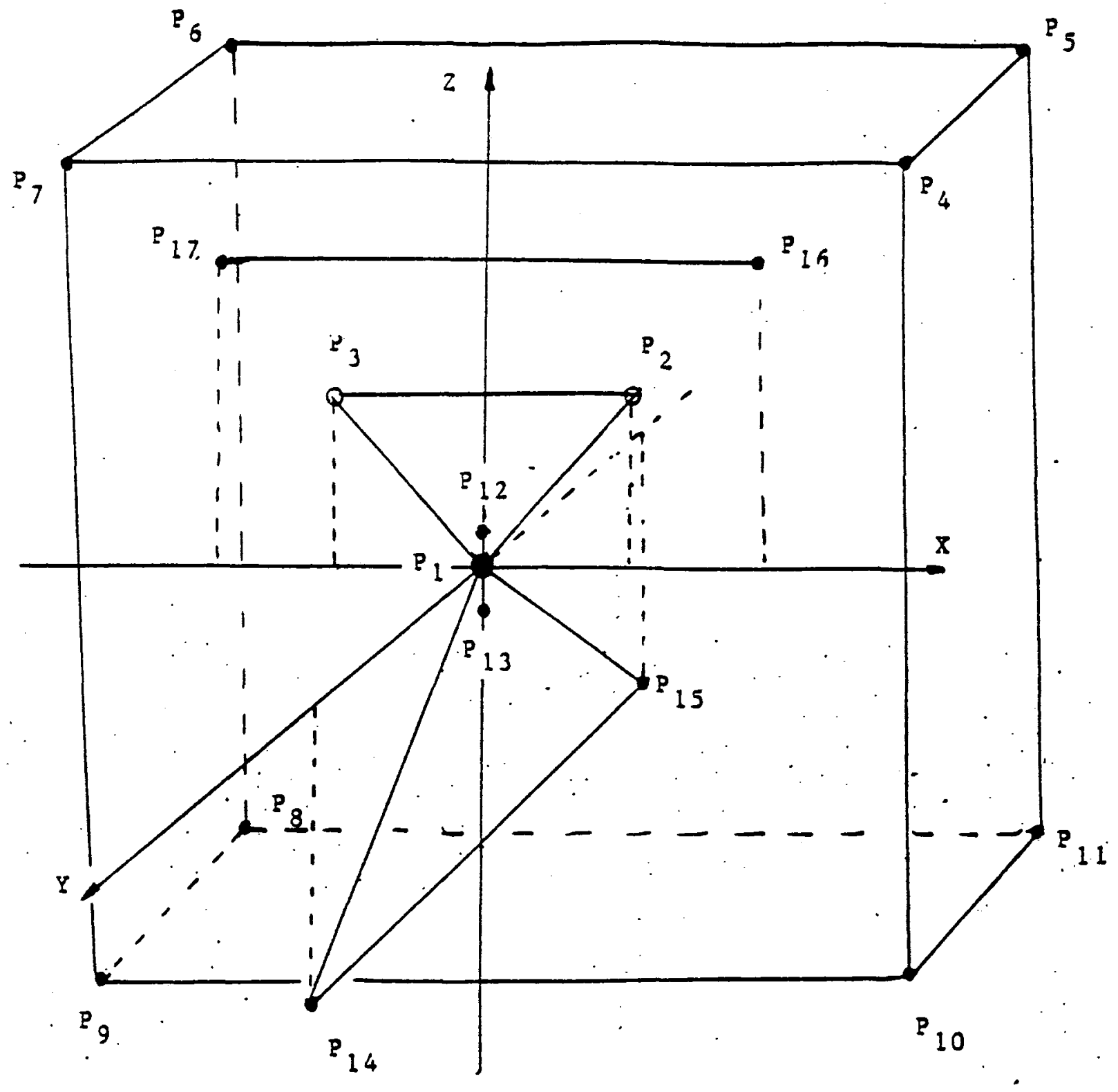

Figure 3.1 
then system (3.1) simplifies to

(3.12)

$$
M_{i} \frac{d^{2} \vec{R}_{i}}{d T^{2}}=(23.06689) \sum_{\substack{j=1 \\ j \neq i}}^{17} \frac{E_{i} E_{j}}{R_{i j}^{2}} \frac{\vec{R}_{j i}}{R_{i j}}, i=2,3, \ldots, 13
$$

in which

$$
\begin{aligned}
& M_{1}=16(16724) \\
& M_{2}=M_{3}=16724 \\
& M_{4}=M_{5}=\ldots=M_{12}=M_{13}=9.1085 \\
& E_{1}=8 \\
& E_{2}=E_{3}=1 \\
& E_{4}=E_{5}=\ldots=E_{13}=-1 \\
& E_{14}=E_{15}=0.45 \\
& E_{16}=E_{17}=-0.23
\end{aligned}
$$

Further, energy equation ( 3.10$)$ reduces to

$$
-(3333.04) 10^{-4}=\frac{1}{2} \sum_{i=2}^{13} M_{i} V_{i}^{2}+23.06689 \sum_{j=i+1}^{17} \sum_{i=1}^{16} \frac{E_{i} E_{j}}{R_{i j}}
$$

Unless otherwise specified, further discussion is in terms of the $R$ and $T$ variables.

At this point we introduce electron pairing by means of a new model of bonding which has thus far yielded the correct bond lengths and bond angles for dynamical simulations of all the diatomic molecules through oxygen ${ }^{(14)}$. We assume that the hydrogen electrons bond with two oxygen electrons through electron attraction.

Observe that electron attraction is, of course, not unknown. For example, Nobelists Bardeen, Cooper, and Schrieffer showed that the quantum mechanical theory of superconductivity requires electron attraction ${ }^{(15)}$. In addition, it must be noted that the behavior of electrons and protons within atoms and molecules has not been determined experimentally. Pauling and Wilson point out that "We do not know that the electron and proton attract each other in the same way that two macroscopic electrically charged bodies do, inasmuch as the force between two particles in a hydrogen atom has never been directly measured" (16).

For consistency with previous studies we choose the paired electrons to be separated maximally, so the pairs $\left(P_{4}, P_{8}\right),\left(P_{6}, P_{10}\right)$ will be chosen. We must then replace the terms $E_{4} E_{8}$ and $E_{6} E_{10}$ in both (3.12) and (3.13) by $-E_{4} E_{8},-E_{6} E_{10}$, respectively. The new equations will be called the semiclassical equations.

Once initial data are assigned, it will be incumbent upon us to solve semiclassical system (3.12) in such a fashion that the energy at each time step is invariant, that is, that 
4. Assignment of Initial Data. As shown in Figure 3.1, the initial positions of $P_{1}-P_{17}$, in $(X, Y, Z)$ coordinates, will be set as follows. The arrangement will assure a degree of symmetry which will decrease the required computer time.

$P_{1}$ is fixed for all time at $(0,0,0)$. In terms of positive parameters $X_{2}$ and $Z_{2}, P_{2}$ and $P_{3}$ are set at $\left(X_{2}, 0, Z_{2}\right),\left(-X_{2}, 0, Z_{2}\right)$, respectively, with initial velocities $\overrightarrow{0}$. For positive parameter $R_{1}, P_{4}-P_{11}$ are set, respectively, at $\left(R_{1}, R_{1}, R_{1}\right),\left(R_{1},-R_{1}, R_{1}\right)$, $\left(-R_{1},-R_{1}, R_{1}\right), \quad\left(-R_{1}, R_{1}, R_{1}\right), \quad\left(-R_{1},-R_{1},-R_{1}\right), \quad\left(-R_{1}, R_{1},-R_{1}\right)$, $\left(R_{1}, R_{1},-R_{1}\right),\left(R_{1},-R_{1},-R_{1}\right)$. In terms of a positive velocity parameter $V_{1}$, the initial. velocities of $P_{4}-P_{11}$ are taken, respectively, to be $\left(0,-V_{i}, 0\right),\left(-V_{1}, 0,0\right)$, $\left(0, V_{1}, 0\right),\left(V_{1}, 0,0\right),\left(V_{1}, 0,0\right),\left(0,-V_{1}, 0\right),\left(-V_{1}, 0,0\right),\left(0, V_{1}, 0\right)$. The two first ring electrons $P_{12}$ and $P_{13}$ are set at $(0,0,680),(0,0,-680)$, and since the ground state energy ${ }^{(10)}$ of $0^{6+}$ is $(-1952.9758) 10^{-12}$ erg, their velocities are approximated to be $(0,0.171453,0),(0,-0.171453,0)$, respectively. $P_{14}-P_{17}$ are fixed for all time at $(0,22500,-15900), \quad(0,-22500,-15900), \quad(22500,0,15900), \quad(-22500,0,15900)$, respectively.

Once the parameters $X_{2}, Z_{2}$, and $R_{1}$ are selected, the value $V_{1}$ follows directly from semiclassical (3.13), and all initial data will be determined. Thus, for example, for $X_{2}=7570, Z_{2}=5860, R_{1}=4500$, one finds $V_{1}=0.02420179732$.

5. Examples. Observe next that, whereas water vapor spectra ${ }^{(1)}$ imply that the average bond length of a water molecule is $0.96 \AA$ with an average bond angle of $104.5^{\circ}$, one might expect variations in ice $I$ due to the expansion of water at $4^{\circ} \mathrm{C}$. Indeed, experimental NMR results ${ }^{(1)}$ which relate allowable bond angles and bond lengths for ice are those which lie in the shaded region of Figure 5.1. Thus, for example, for an angle of $104.5^{\circ}$, a bond length of $0.99 \stackrel{\circ}{\AA}$ would be consistent with NMR measurements, while a bond length of $0.96 \AA$ would not.

Figures 5.1 and 2.3 now lead to the following surmises. Initial data which lie in the stable shaded region of Figure 5.1 should yield dynamical results which also lie in the stable region, since the effects of $C_{1}$ and $C_{2}$ on the protons should be in relative balance. Initial data which lie below the stable region should, in time, yield dynamical results which also lie in the stable region, because the repulsive effects of $C_{1}$ on the protons should dominate. Initial data which lie above the stable region should yield dynamical results which do not lie in the stable region because the attractive effects of $C_{2}$ on the protons should dominate. We will see next through several extensive examples that these surmises are correct. Calculations will be described in terms of the $R$ and $T$ variables discussed in Sections 3 and 4. Each example was run on an Alpha 150 or an Alpha 275 using a 64 bit word. The energy conserving method ${ }^{(17)}$ incorporates iteration tolerances of $4(10)^{-9}$ for position components and $4(10)^{-12}$ for velocity components. A variable time step procedure is incorporated as follows. At each time step, let $R^{*}=\min R_{i j} ; i=1,2, \ldots$, $13 ; j=1,2, \ldots, 13 ; i \neq j$. Then 


$$
\begin{aligned}
& \text { if } \quad 1500 \leq R^{*} \quad \text {, set } \Delta T=2.0 \text {, } \\
& \text { if } \quad 1000 \leq R^{*}<1500 \text {, set } \Delta T=1.0 \text {, } \\
& \text { if } \quad 750 \leq R^{*}<1000 \text {, set } \Delta T=0.2 \text {, } \\
& \text { if } \quad 500 \leq R^{*}<750 \text {, set } \Delta T=0.02 \text {, } \\
& \text { if } \quad 250 \leq R^{*}<500 \text {, set } \Delta T=0.002 \text {, } \\
& \text { if } \quad R^{*}<250 \text {, set } \Delta T=0.0002 \text {. }
\end{aligned}
$$

Ionization, which does occur rarely ${ }^{(1)}$, is neglected by assuming that, on the average, each water molecule has ten electrons. To implement this conservatively, we assume that if $\left|P_{1} P_{2}\right|, j=4,5,6, \ldots, 13$ exceeds 13800 units (i.e., $\frac{1}{2}(2.76) \AA$ ), then $P_{j}$ is reflected simply by resetting its velocity $\vec{V}_{j}$ to $-\vec{V}_{j}$.

A typical FORTRAN program is available in Appendix A of Greenspan ${ }^{(18)}$. A program to generate initial data is available in Appendix B of the same reference.

Consider now the choices $X_{2}=7933.5334, Z_{2}=6087.6143, R_{1}=4500$, for which $V_{1}=0.02423441038$. For these data the $\mathrm{HOH}$ angle is $105^{\circ}$ and the $\mathrm{OH}$ length is 10000 , which are shown at the point $A$ in Figure 5.2. The variation of the bond length with the bond angle is shown in the figure through three billion time steps and terminates at $A^{\prime}$. The real time of the system's motion is $(5.18) 10^{-16} \mathrm{sec}$. Though two electrons were always core electrons, these were not always the same two, which is consistent with recent quantum Monte Carlo simulations ${ }^{(19)}$.

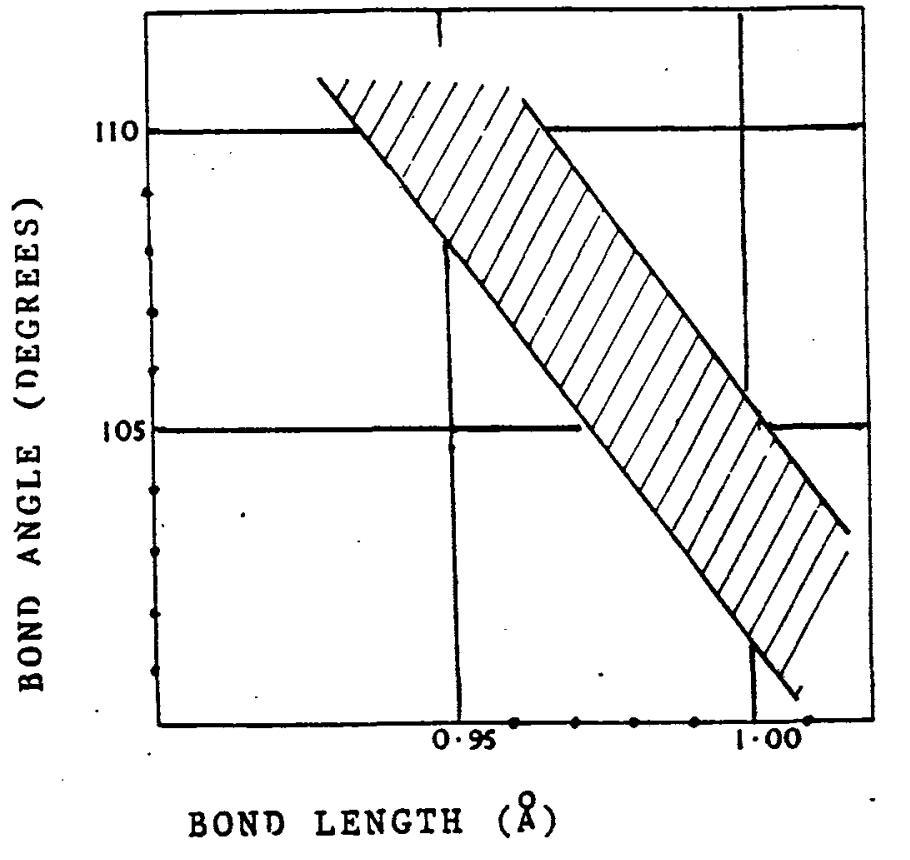

Figure 5.1. Ice $I$ equilibrium dimensions derived from NMR measurements and shown as a shaded strip. 
Consider second the choices $X_{2}=7570, Z_{2}=5860, R_{1}=4500$, so that $V_{1}=0.02420179732$. For these data, the angle $\mathrm{HOH}$ is $104.5125^{\circ}$ and the $\mathrm{OH}$ length is 9573.1134, which are shown as the point $B$ in Figure 5.2. The variation of bond length with bond angle is shown in the figure through four billion time steps and terminates at $B^{\prime}$. The related real time is (4.99) $10^{-16}$ sec. The initial point $B$ is outside and below the allowable NMR region, but the terminal point $B^{\prime}$ is inside.

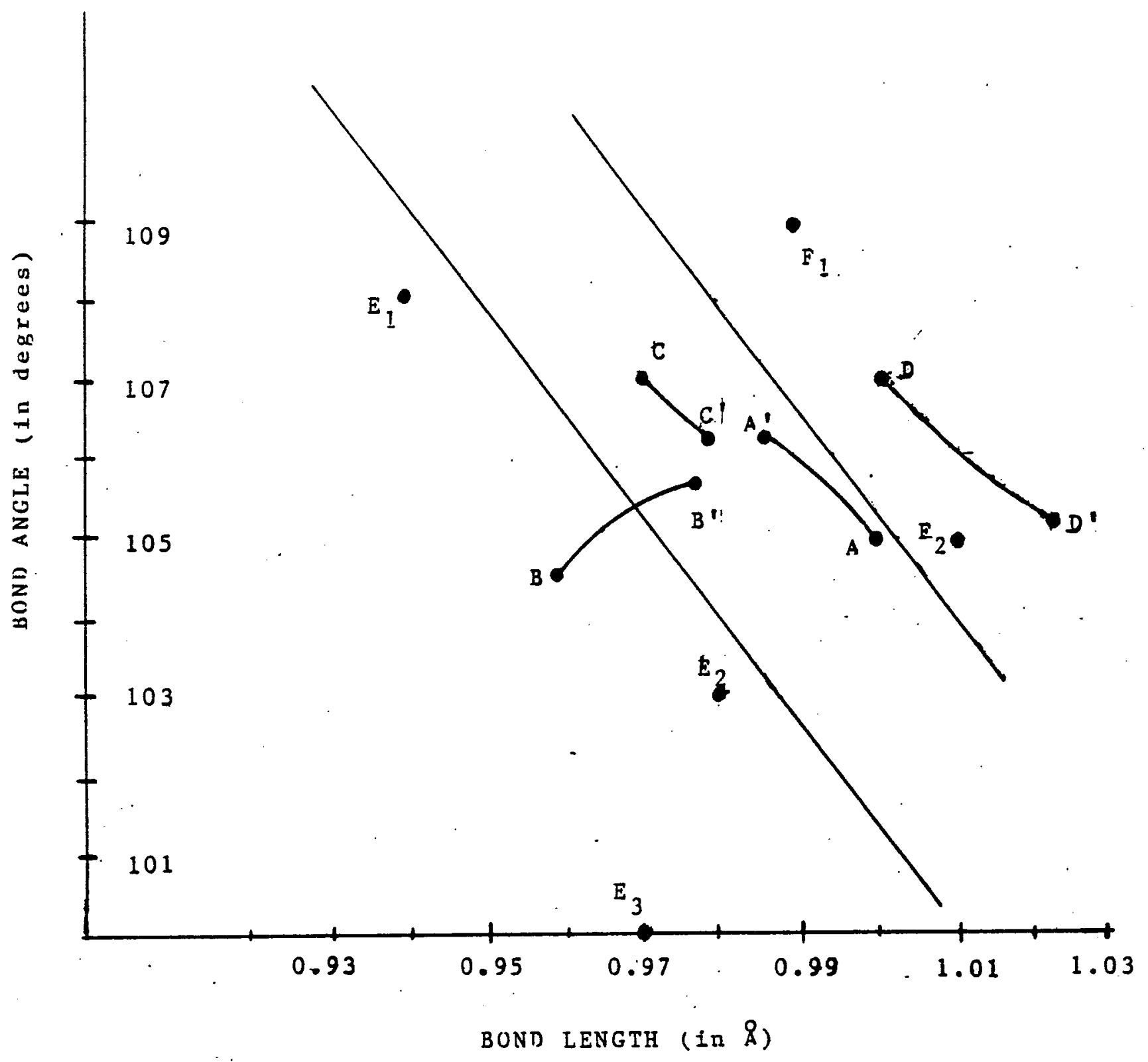

Figure 5.2 
For the third example, consider $X_{2}=7797.4115, Z_{2}=5769.7810, R_{1}=4500$, so that $V_{1}=0.02419214137$. For these data, the angle $\mathrm{HOH}$ is $107^{\circ}$ and the length $\mathrm{OH}$ is 9700 , which are shown as the point $C$ in Figure 5.2. The point $C$ is relatively high in the shaded area of Figure 5.1. The variation of bond length with bond angle is shown in Figure 5.2 through three billion time steps and terminates at $C^{\prime}$. The real time of the system's motion is $(3.83) 10^{-16}$ sec. The results are entirely within the acceptable NMR region.

Consider next $X_{2}=8038.5686, Z_{2}=5948.2279 ; R_{1}=4500$, so that $V_{1}=0.02421523072$. For these data, the angle $\mathrm{HOH}$ is $107^{\circ}$ and the length $\mathrm{OH}$ is 10000 , which are shown as the point $D$ in Figure 5.2. The point $D$ is above the allowable NMR region. The variation of bond length with bond angle is shown in Figure 5.2 through 2.1 billion time steps and terminates at $D^{\prime}$. The related real time is (3.94)10 $10^{-16}$ sec. The motion is clearly away from the stable NMR region and is characterized in particular by rapidly increasing values of $\mathrm{OH}$.

For $R_{1}=4500$, additional examples with initial data $E_{1}, E_{2}, E_{3}$ below the allowable NMR region (consult Figure 5.2) and with initial data $F_{1}, F_{2}$ above the NMR region were also run, but only until their larger motions were apparent. For the cases $E_{1}$, $E_{2}, E_{3}$ the motion was toward the NMR region while for $F_{1}, F_{2}$ the motion was away from the stable region.

All the examples in this section confirm the surmises made at the very beginning of this section.

6. Remarks. Some additional important points must now be made.

It was shown previously that pairing is essential for a semiclassical, dynamical simulation of $\mathrm{H}_{2}{ }^{(20)}$. To test the importance of pairing in the present study, we ran the second example of Section 5 but without pairing for two billion time steps. Whereas with pairing the bond length varied with the bond angle from $B$ to $B^{\prime}$ in Figure 5.2, without pairing there was no such variation at all. Indeed, while the angle remained at $104.5^{\circ}$ throughout the simulation, the bond length decreased from 9573 to 9570 .

Replacement of the Duncan and Pople constants $0.45 e,-0.23 e$ by the Bjerrum ${ }^{(7)}$ constants $0.53 e,-0.23 e$ or by the Rowlinson ${ }^{(6)}$ constants $0.38 e,-0.23 e$ had only minor effects on dynamical results.

Finally, replacement of the reflection from a sphere to reflection from a cube with edge length 27600 and $P_{1}$ at its center also had only a minor effect on dynamical results.

\section{REFERENCES}

1. D. Eisenberg and W. Kauzmann, The Structure and Properties of Water, Oxford Univ. Press, Oxford, 1969.

2. R. A. Horne (Ed.), Water and Aqueous Solutions: Structure, Thermodynamics, and 
Transport Processes, Wiley, N.Y., 1972.

3. R. A. Horne, Encyclopedia Brittanica, $15^{\text {th }}$ Ed., Vol 15, 1993, p. 759.

4. I. Ohmine, J. Chem. Phys., 99, 1995, p. 6767.

5. R. J. Gillespie, J. Chem. Ed., 40, 1963, p. 295.

6. J. Hirschfelder, D. F. Curtiss, and R. B. Bird, Molecular Theory of Gases and Liquids, Wiley, N. Y., 1967.

7. N. Bjerrum, Science, 115, 1952, p. 385.

8. L. Pauling, The Nature of the Chemical Bond, Cornell Univ. Press, $3^{\text {rd }}$ Ed., Ithaca, 1973.

9. B. H. Mahan, University Chemistry, Addison-Wesley, Reading, MA, 1966.

10. D. R. Lide (Ed.), CRC Handbook of Chemistry and Physics, CRC Press, Boca Raton, FL, 1990-1991.

11. D. Park, Introduction to Quantum Theory, McGraw-Hill, N.Y., 1964.

12. M. Gell-Mann and J. B. Hartle, UCSBTH - 91 - 15, UC Santa Barbara (no date).

13. M. Nauenberg, C. Stroud and J. Yeazell, Scientific American, June, 1994, p. 44.

14. D. Greenspan, Physica Scripta, 52, 1995, p. 267.

15. J. Bardeen, L. N. Cooper, and J. R. Schrieffer, Phys. Rev., 108, 1957, p. 1175.

16. L. Pauling and E. B. Wilson, Introduction to Quantum Mechanics, Dover, N.Y., 1963.

17. D. Greenspan, Comp. Math. Applic., 29, 1995, p. 37.

18. D. Greenspan, TR\#303, Math Dept., UT Arlington, Arlington, TX USA 76019.

19. Z. Sun, M. M. Soto, and W. A. Lester, J. Chem. Phys., 100, 1994, p. 1278.

20. D. Greenspan, Comp. Math. Applic., 22, 1991, p. 73. 


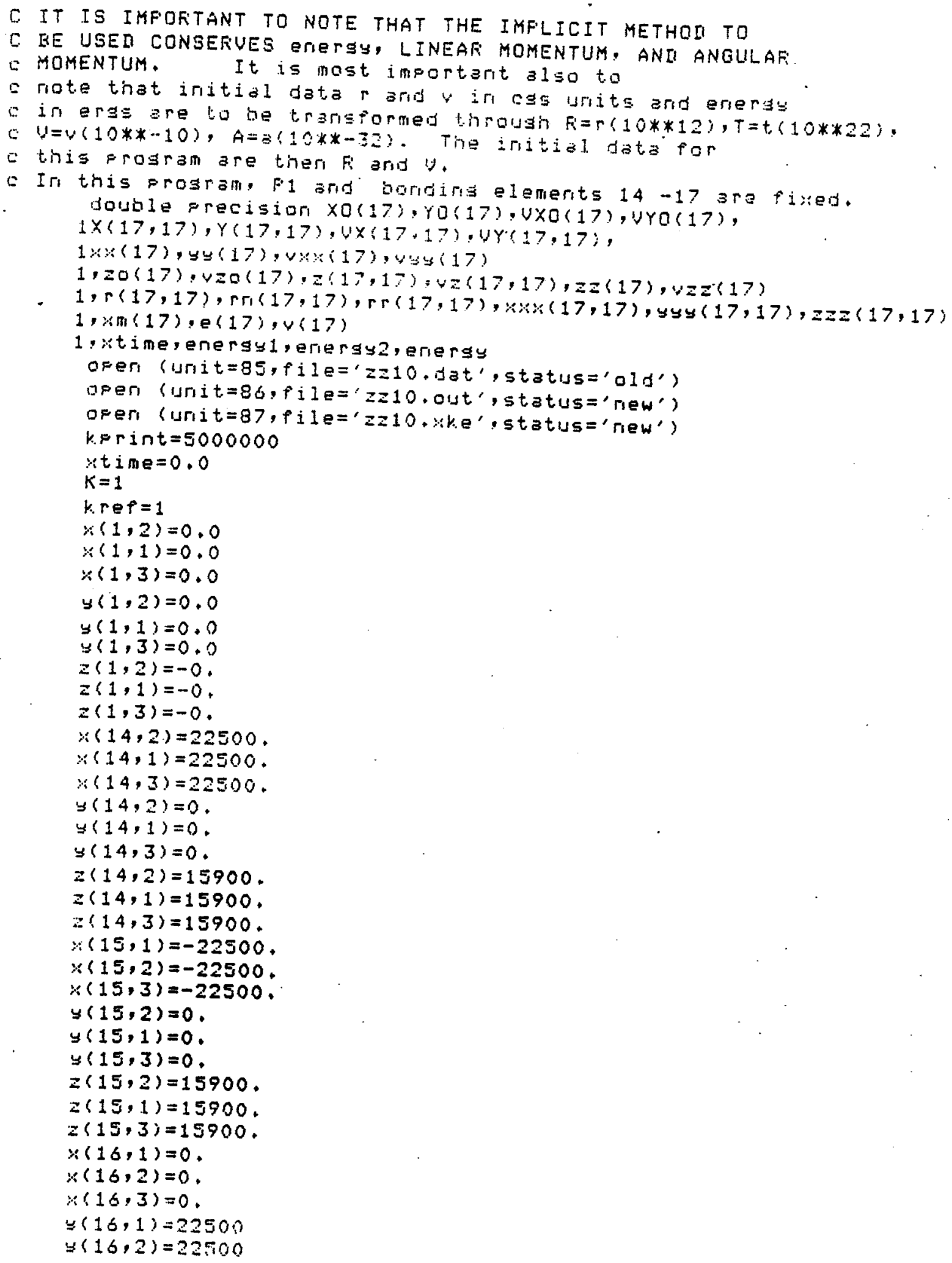




$$
\begin{aligned}
& \checkmark(16,3)=22500 \\
& z(16,1)=-15900 \text {. } \\
& z(16,2)=-15900 \text {. } \\
& z(16,3)=-15900 \text {. } \\
& \because(17,1)=0 \text {. } \\
& x(17,2)=0 \text {. } \\
& x(17,3)=0 \text {. } \\
& y(17,1)=-22500 \text {. } \\
& \exists(17,2)=-22500 \text {. } \\
& y(17,3)=-22500 \text {. } \\
& z(17,1)=-15900 \text {. } \\
& z(17,2)=-15900 \text {. } \\
& Z(17,3)=-15900 \text {. } \\
& v \times(1,2)=0.0 \\
& v x(1,1)=0.0 \\
& w x(1,3)=0.0 \\
& v y(1,2)=0 \text {. } \\
& \forall y(1,1)=0 \text {. } \\
& v_{Y}(1,3)=0 \text {. } \\
& v \geq(1,2)=-0 \text {. } \\
& v=(1,1)=-0 \text {. } \\
& v z(1,3)=-0 \text {. } \\
& v \times(14,1)=0.0 \\
& v \times(14,2)=0.0 \\
& v x(14,3)=0.0 \\
& V y(14,2)=-0 \text {. } \\
& \checkmark y(14,1)=-0 \text {. } \\
& \text { yy }(14,3)=-0 \text {. } \\
& v \geq(14,2)=-0 \text {. } \\
& v z(14,1)=-0 \text {. } \\
& v z(14,3)=-0 \text {. } \\
& v \times(15,1)=0.0 \\
& v \times(15,2)=0.0 \\
& w \times(15,3)=0.0 \\
& \forall y(15,2)=-0 \text {. } \\
& v_{S}(15,1)=\cdots 0 \text {. } \\
& v y(15,3)=-0 \text {, } \\
& v=(15,2)=\cdots 0 \text {. } \\
& y \geq(15,1)=-0 \text {. } \\
& v=(15,3)=-0 \text {. } \\
& v \times(16,2)=0.0 \\
& v \times(16,1)=0.0 \\
& v \times(16,3)=0.0 \\
& v_{Y}(16,2)=0 \text {. } \\
& y y(16,1)=0 \text {. } \\
& y y(16,3)=0 \text {. } \\
& y=(16,2)=-0 \text {. } \\
& v=(16,1)=-0 \text {. } \\
& y=(16,3)=-0 \text {. } \\
& w(17,1)=0.0 \\
& v \times(17,2)=0.0 \\
& Y \times(17,3)=0.0 \\
& v y(17,2)=-0 \text {. } \\
& \checkmark y(17,1)=-0 \text {. } \\
& v_{y}(17,3)=-0 \text {. } \\
& v z(17,2)=-0 \text {. } \\
& v z(17,1)=-0 \text {. } \\
& v z(1.7,3)=-0 \text {. }
\end{aligned}
$$


C FEAII IN THE INITTAL IIATA.

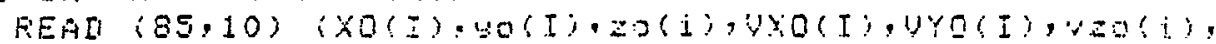
$1 i=1,13$ )

10 FOFMAT $(3 f 16.8, f 16.11,2 f 16.11)$

C WE NOW SOLVE FOR THE NEW FOSITIONS ANI UELOCITIES WITH

C. FOFMULAS WHICH FIEQUIFE ITEFATION, WE WILL ISE THE

I IIIEAS OF THE GENERALIZEI NEWTON'S METHOD WIthOI:

\& urdetina esch iterate.

$c$

C SET THE INFUT IIATA.

I0 $30 \quad i=2,13$

$X(I, 1)=X 0(I:$

$Y(I, 1)=Y O(I ;$

$Z(I, I)=Z 0(I)$

$U \times(I, 1)=U \times 0(I)$

$U Y(I, 1)=U Y C(I$ )

$V=(I, 1)=U=0(I)$

30 CONTINUE

GO TO 100

$65 \quad$ I10 $70 \quad \mathrm{i}=2.13$

$X(I, 1)=X(I, 2)$

$Y(I, 1)=Y(I, 2)$

$I(I, 1)=I(I, 2)$

$U \times(I, 1)=U X(I, 2)$

$U Y(I, 1)=U Y(I, 2)$

$V Z(I, 1)=U Z(I, 2)$

70 . CONTINUE

C FIX THE FIRST GUESS $X(I, 2)$ OF THE ITERATION.

$100 \quad 0101633 \quad i=2,13$

$X(I, 2)=X(I, 1)$

$Y(I, 2)=Y(I, 1)$

$Z(I, 2)=I(I, 1)$

$U X(I, 2)=U X(I, 1)$

$U Y(I, 2)=U Y(I, 1)$

$U \equiv(I, 2)=U I(I, 1)$

1633 CDNTINUE

do $103 i=1: 17$

do $102 j=1,17$

$r(i, j)=(x(i, 1)-k(j, 1)) * * 2+(y(i, 1)-y i j, 1)) * * 2$

$1+(z(i, 1)-z(j, 1)) * * 2$

102

$r(i, j)=d \operatorname{art}(r(i, j))$

103 coritiriue

continue

de $1 t=2,0$

$\mathrm{min}=100000$.

so $1111 i=1,12$

k. k. $k=i+1$

do $1112, j=k, k, 13$

if $(r(i, j), 1 t, m i n) m i n=r(i, j)$

1112 continuse

1111 contirise 


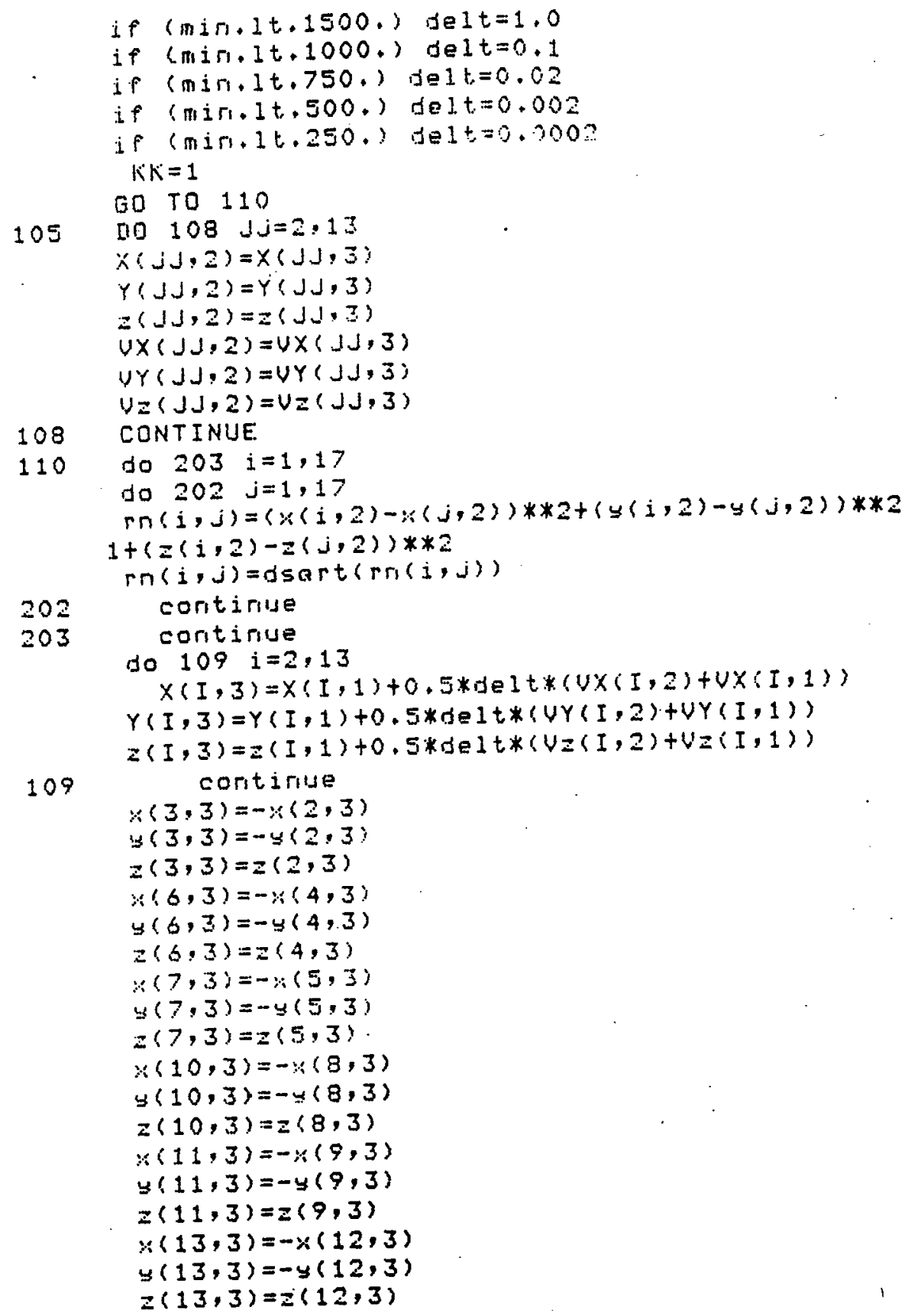


Jo $300 \quad i=1,17$

to $209 \mathrm{j}=1,1, \mathrm{i}$

$x \times x(i, j)=x(i, 2)+\cdots(i, 1)-x(j, 2)-\cdots(j, 1)$

$\checkmark y y(i, j)=y(i, 2)+y(i, 1)-y(j, 2)-y(j, 1)$

299

$z \Sigma z(i, j)=z(i, 2)+z(i, j)-z(j, 2)-z(j, 1)$

continuse

300

continue

do $400 \quad i=1,17$

so $399 j=1,17$

$r r(i, j)=r(i, j) * r n(i, j) *(r i j, j)+r n(i, j))$

399

contirise

400

continue

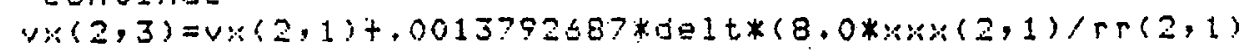

$1+x \times x(2,3) / \operatorname{rr}(2,3)-x \times(2,4) / \operatorname{rr}(2,4)$

$1-x \times x(2,5) / \operatorname{rr}(2,5)-\cdots x(2,6) / r r(2,6)$

$1-x \times x(2,7) \operatorname{rr}(2,7)-x \times x(2,8),(r r(2,8)$

$1-x x x(2,9) / r r(2,9)-x \times x(2,10) / r r(2,10)$

$1-x \cdots \times(2,11) / \operatorname{rr}(2,11)-x \cdots \times(2,12) / \operatorname{rr}(2,12)$

$1-x \times x(2,13) / r r(2,13)-.23 * \cdots x \times(2,14) / r r(2,14)$

$1-.23 * x \cdots \times(2,15) / r r(2,15)+.45 * x \times \cdots(2,16) / r r(2,16)$

$1+.45 * \cdots \cdots \times(2,17) / \mathrm{rr}(2,17))$

$v_{y}(2,3)=v y(2,1)+.0013792687 *$ delt* $(8.0 *$ yy $(2,1) / r r(2,1)$

$1+y y y(2,3) / r r(2,3)-y y y(2,4) / r r(2,4)$

$1-y y y(2,5) / r r(2,5)-y y y(2,6) / r r(2,6)$

$1-y y y(2,7) / \operatorname{rr}(2,7)-$ syy $(2,8) / \operatorname{rr}(2,8)$

$1-y y y(2,9) / r r(2,9)-y y y(2,10) / r r(2,10)$

$1-y y y(2,11) / \operatorname{rr}(2,11)-y y y(2 ; 12) / \operatorname{rr}(2,12)$

$1-y y y(2,13) / r r(2,13)-.23 * y y y(2,14) / r r(2,14)$

$1-.23 * y y y(2,15) / r r(2,15)+.45 * y y y(2,16) / r r(2,16)$

$1+.45 * y y y(2,17) / r r(2,17))$

$v z(2,3)=v z(2,1)+.0013792687 *$ de $1 t *(8.0 * z z z(2,1) / r r(2,1)$

$1+z z z(2,3) / r r(2,3)-z z z(2,4) / r r(2,4)$

$1-z z z(2,5) / r r(2,5)-z z z(2,6) / p r(2,6)$

$1-z z=(2,7) / r r(2,7)-z z z(2,8) / r r(2,8)$

$1-z z z(2,9) / r r(2,9)-z z z(2,10) / r r(2,10)$

$1-z \Sigma z(2,11) / r r(2,11)-z z z(2,12) / r r(2,12)$

$1-z z z(2,13) / \operatorname{rr}(2,13)-.23 * z z 2(2,14) / \operatorname{r}(2,14)$

$1-.23 * z=z(2,15) / r r(2,15)+.45 * z z=(2,16) / r r(2,16)$

$1+.45 * z=z(2,17) / r r(2,17))$

$v *(4,3)=v *(4,1)+2.532457594 * \sigma e 1 t *(-3,0 * x * x(4,1) / r r(4,1)$

$1-x x \times(4,2) / \operatorname{rr}(4,2)-x \times x(4,3) / \operatorname{rr}(4,3)$

$1+x x \times(4,5) / r r(4,5)+x x \times(4,6) / r r(4,6)$

$1+\ldots x i(4,7) / \operatorname{rr}(4,7)-x \times(4,8) / \operatorname{rr}(4,8)$

$1+x \times x(4,9) / r r(4,9)+x x x(4,10) / r r(4,10)$

$1+x x \times(4,11) / \operatorname{rr}(4,11)+x \times x(4,12) \operatorname{rr}(4,12)$

$1+x \times x(4,13) / r r(4,13)+.23 * \cdots \cdots \times(4,14) / r r(4,14)$

$1+.23 * x \times \cdots(4,15) / r r(4,15)-.45 * x \times x(4,16) / r r(4.16)$

$1-.45 * \times \times \times(4,17) / r r(4: 17))$

$v y(4,3)=v y(4,1)+2.532457594 * d e 1 t *(-8.0 * y y y(4,1) / r r(4,1)$

$1-s_{y}(4,2) / \operatorname{rr}(4,2)-y_{y}(4,3) / \operatorname{rr}(4,3)$

$1+y y z(4,5) / \operatorname{rr}(4,5)+y y y(4,6) / \operatorname{rr}(4,6)$

$1+y y y(4,7) / \operatorname{rr}(4,7)-y y y(4,8) / \operatorname{rr}(4,8)$

$1+y y y(4,9) / r r(4,9)+y y y(4,10) / r r(4,10)$

$1+y s y(4,11) / \Gamma r(4,11)+s y s(4,12) / r r(4,12)$

$1+y z y(4,13) / r r(4,13)+.23 * y y y(4,14) / r r(4,14)$

$1+.23 *$ yys $(4,15) / \operatorname{rr}(4,15)-.45 *$ yyy $(4,16) / \operatorname{rr}(4,16)$

$1-.45 * y y y(4,17) / r r(4,17))$ 


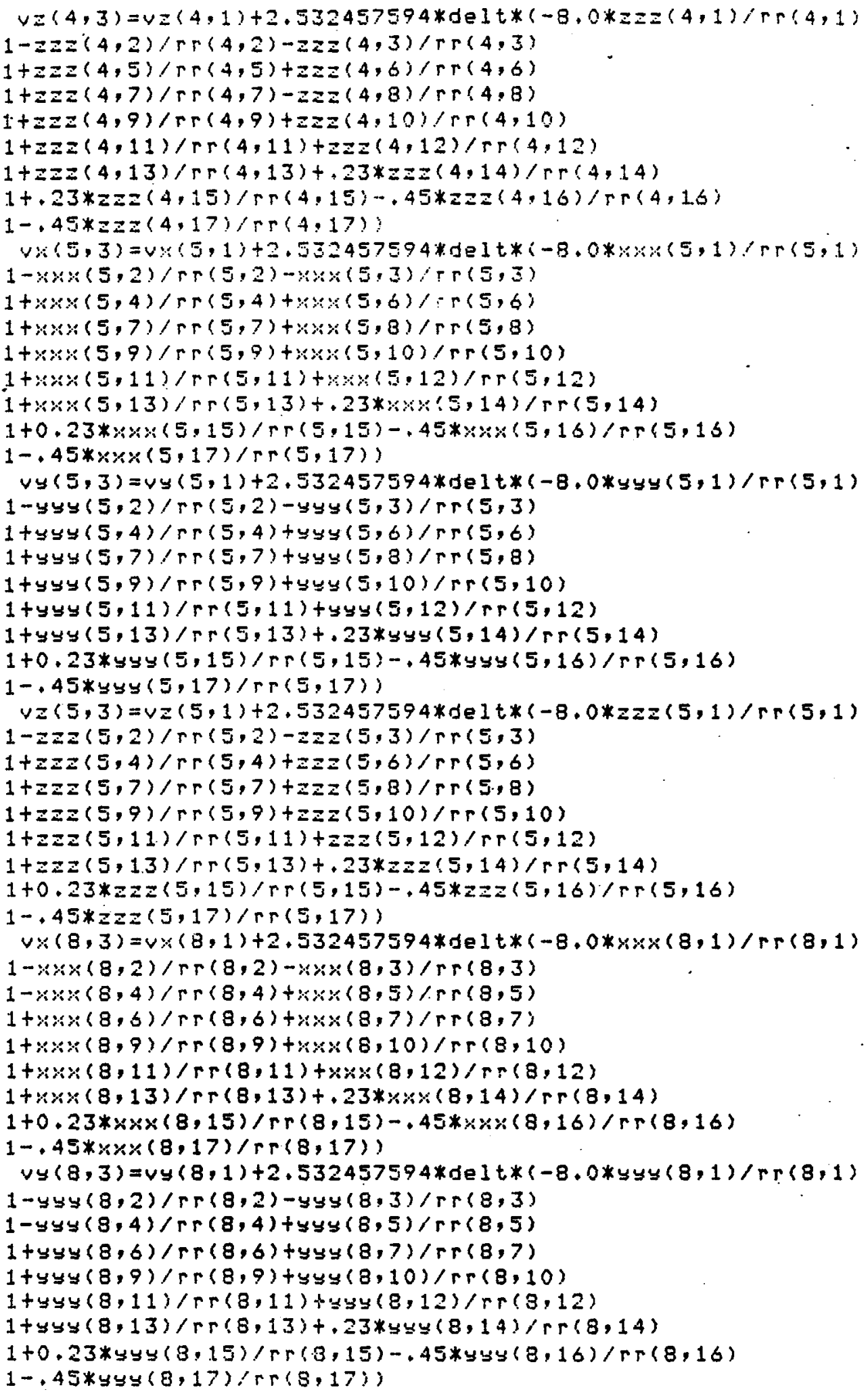




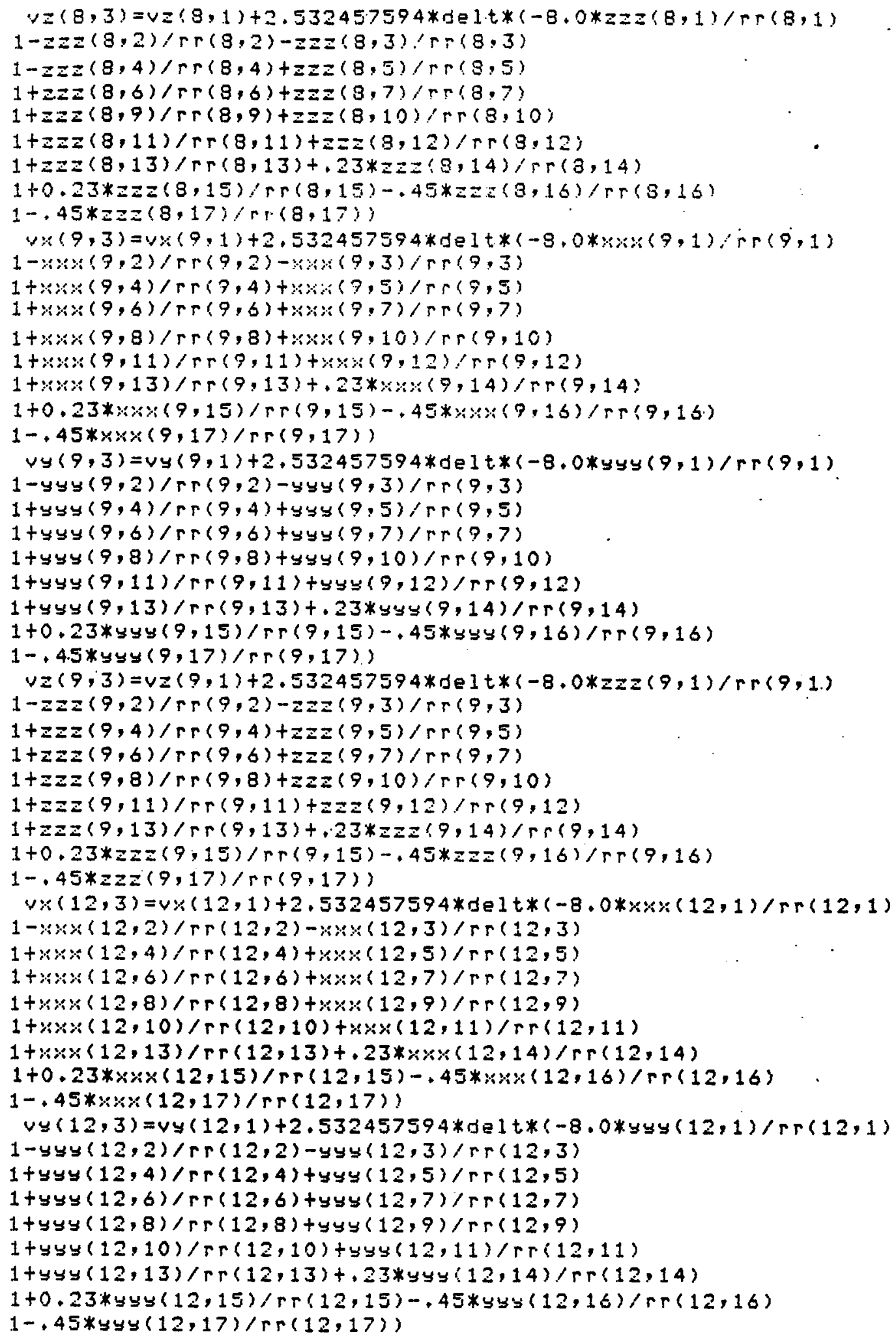




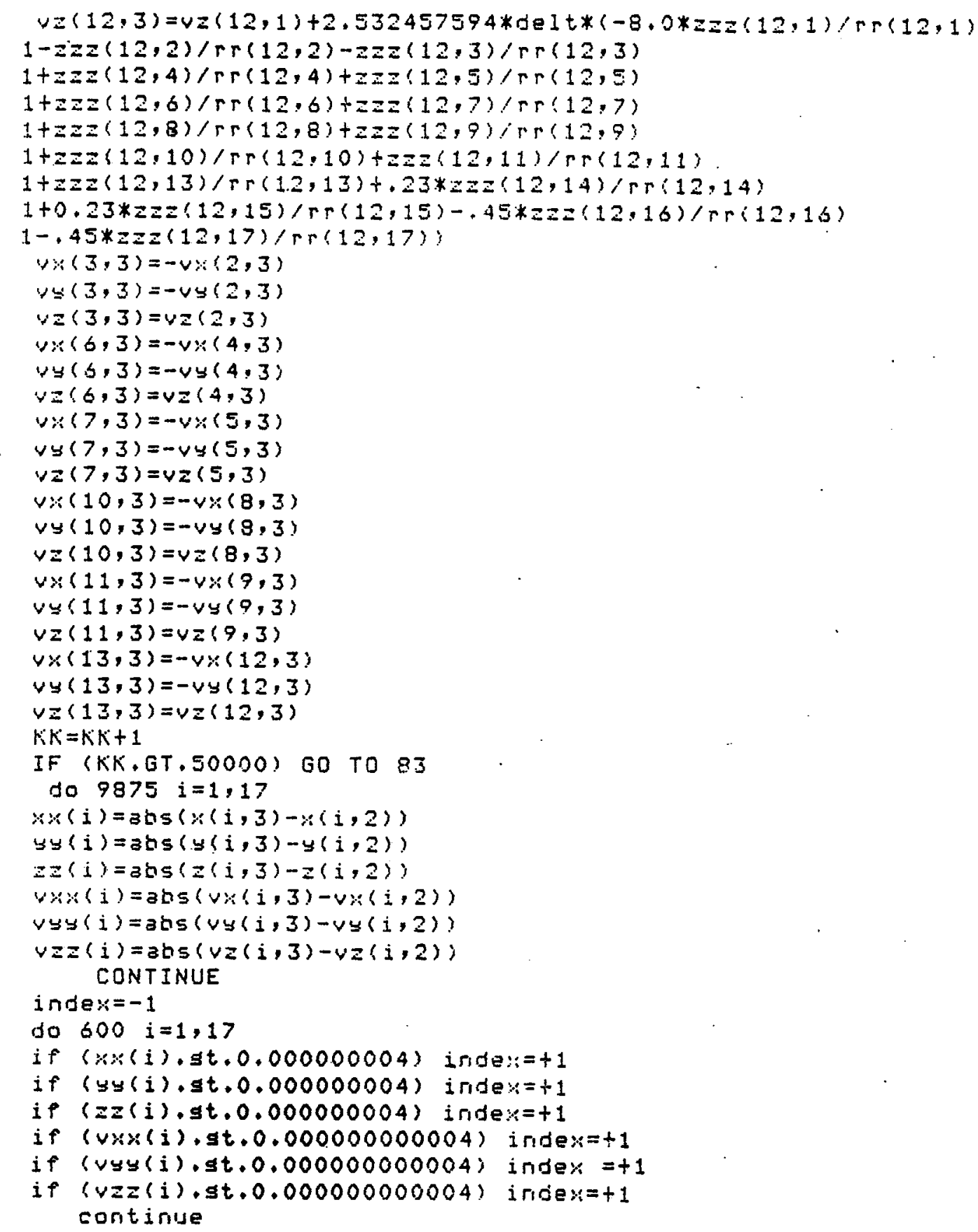




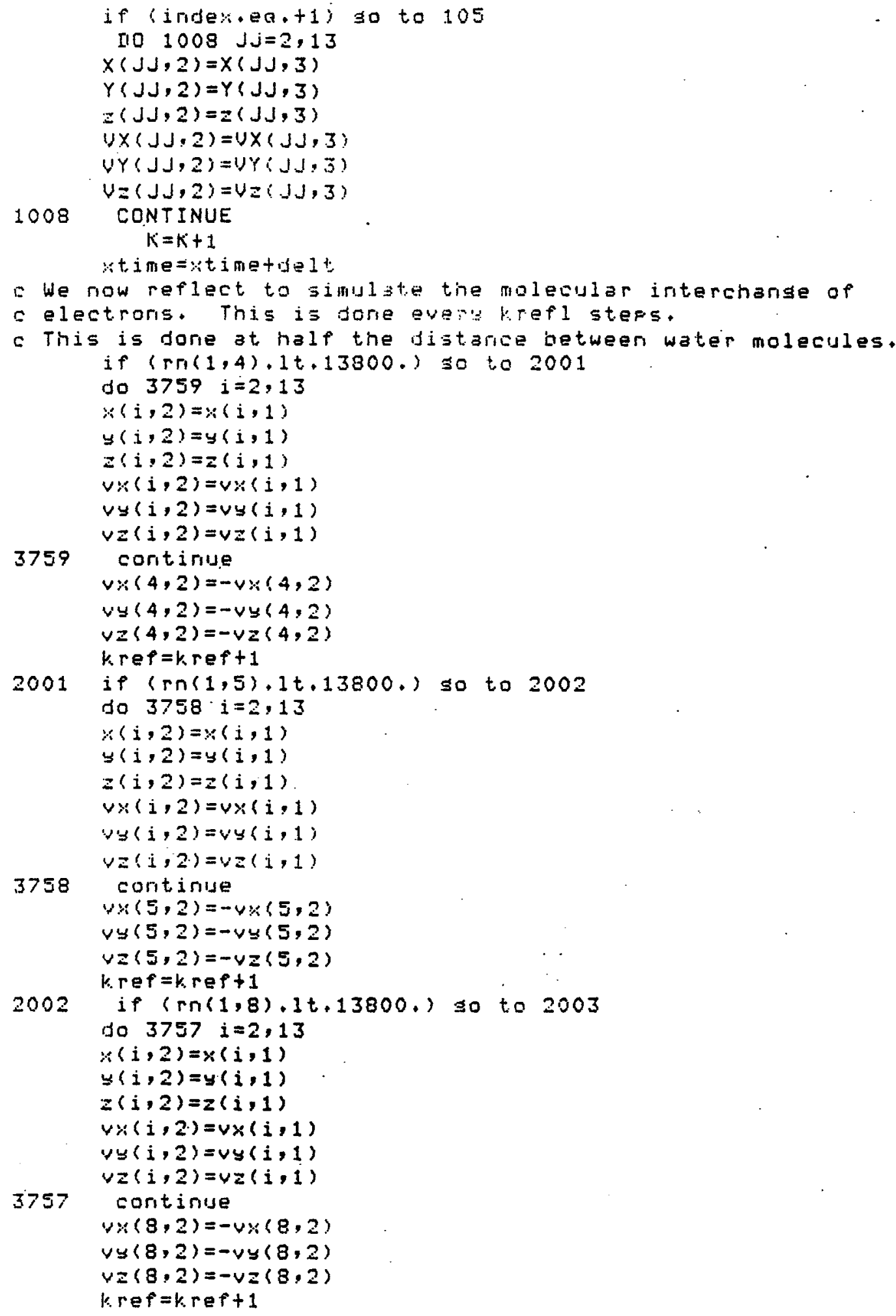




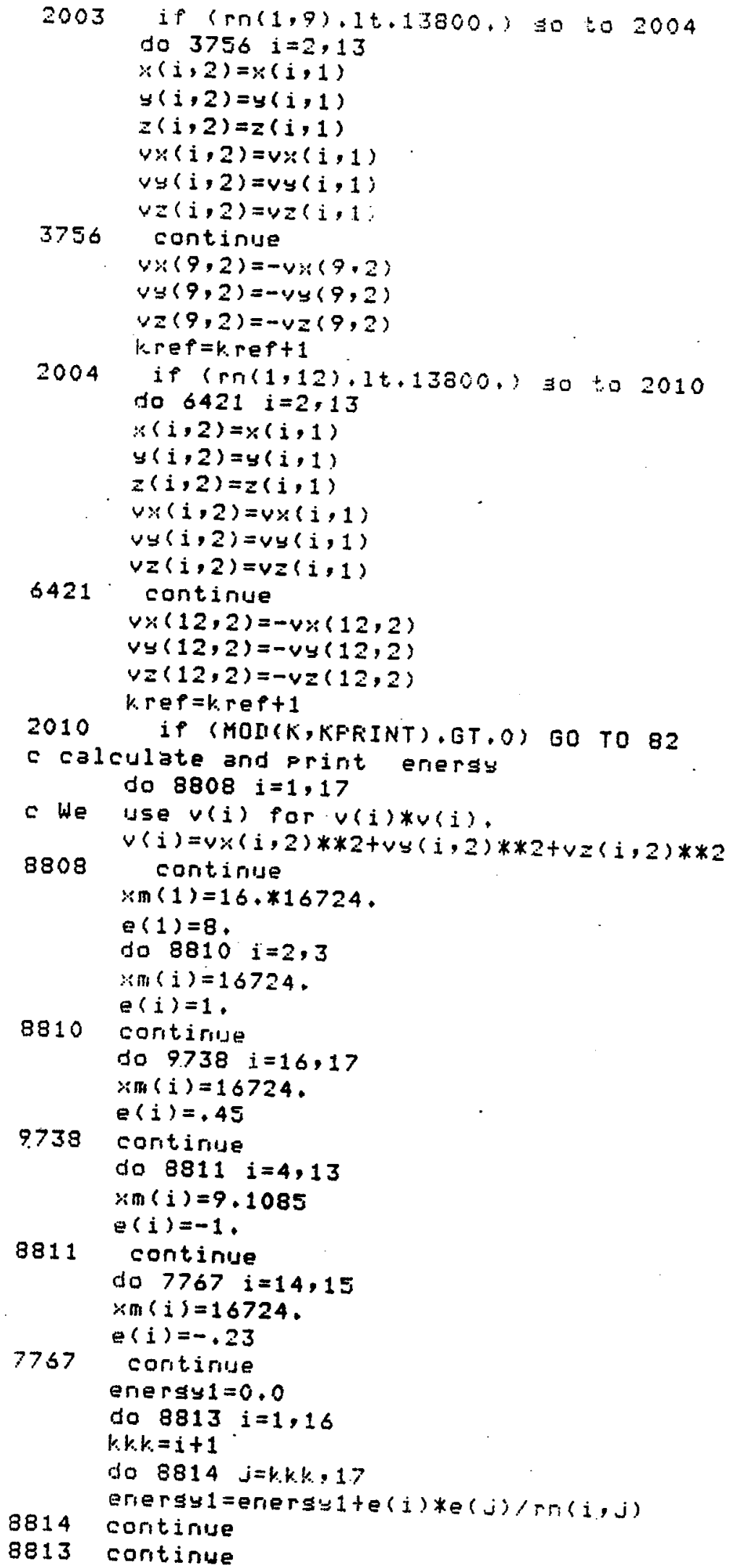




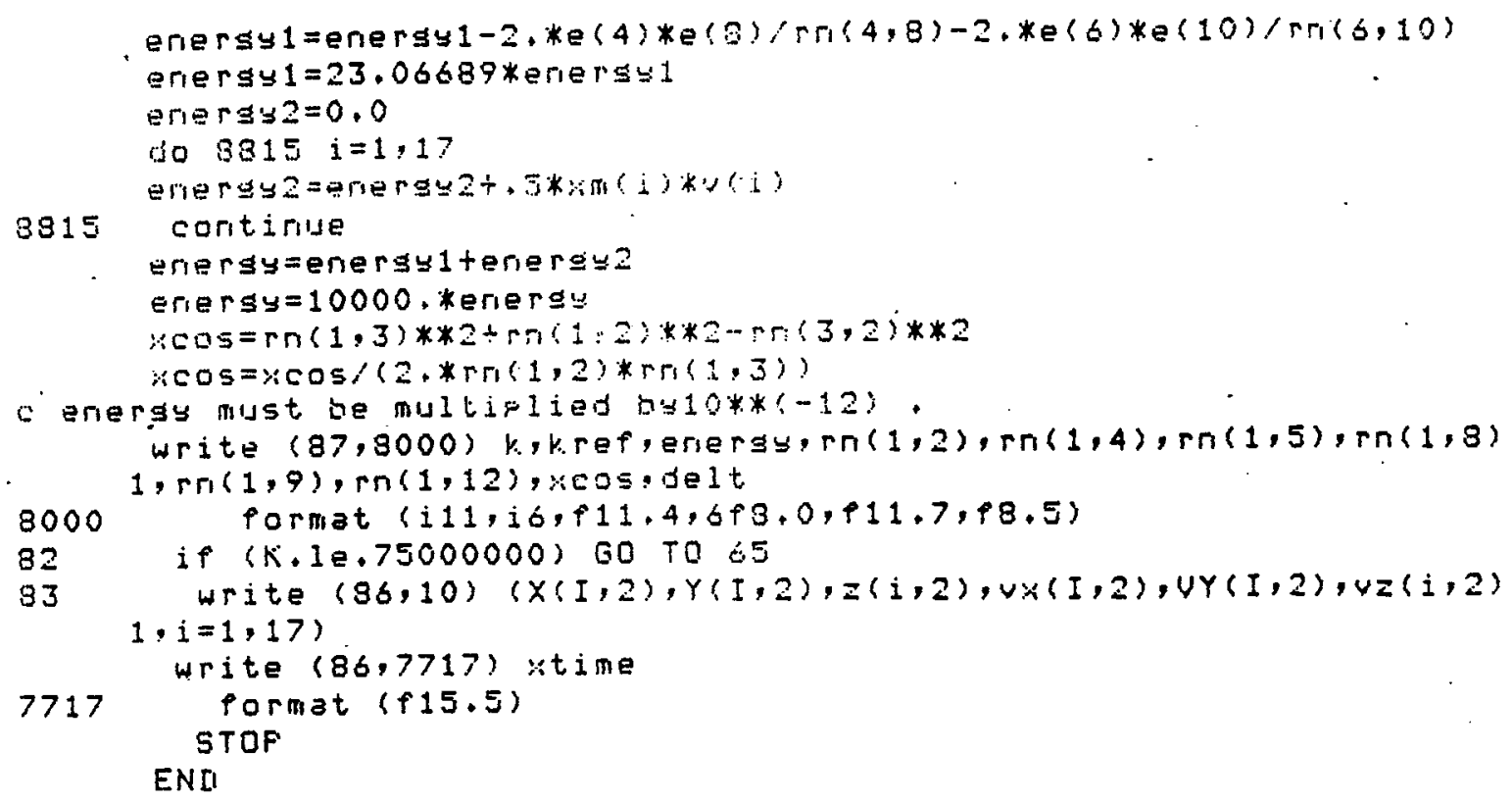




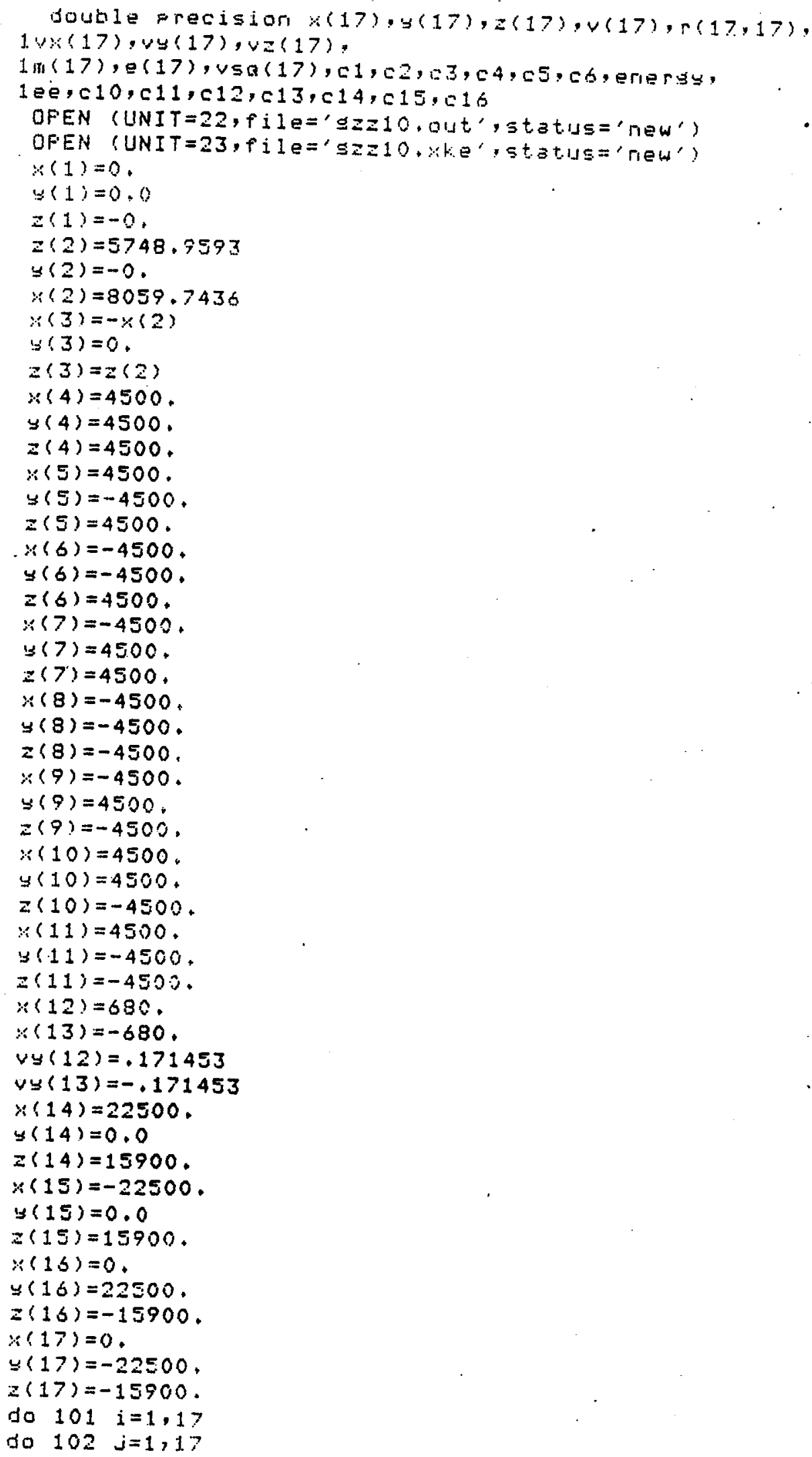




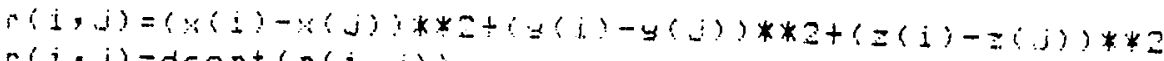

ENII 